\title{
New Recovery Error Indicator for Adaptive Finite-Element Analysis on Waveguiding Structures
}

\author{
Alejandro B. Díaz-Morcillo, Member, IEEE, Juan V. Balbastre, Member, IEEE, and Luis Nuño, Member, IEEE
}

\begin{abstract}
The adaptive finite-element method (FEM) is an iterative variant of the FEM where, in a first step, an initial mesh with few and low-order elements is generated, the corresponding algebraic problem is solved and the error in the solution is estimated in order to add degrees of freedom in those regions of the domain with the biggest error estimation. This process is repeated until an ending condition is reached. The two basic stages in this method are the error indication and the mesh enrichment. In this paper, within the analysis of waveguiding structures, a new error indicator based on the curl recovery is described. In addition, an overview on refinement techniques is presented, and the $h$-refinement employed in this study is briefly described. Results obtained with the curl-recovery indicator are discussed and compared with the classical nonadaptive FEM and two previously developed error indicators: the residual and flux continuity indicators.
\end{abstract}

Index Terms-Error analysis, finite-element methods (FEMs), transmission lines, waveguides.

\section{INTRODUCTION}

$\mathbf{I}$ $\mathrm{N}$ THE finite-element method (FEM), the stage of the geometry discretization has a great importance because the accuracy of the solution is directly related to the size, shape, and order of the elements obtained in the meshing process. In some problems, that solution is smooth enough and high accuracy can be obtained by means of uniform discretizations of the domain. This is the case, for instance, of the analysis of the different modes in a rectangular and homogeneous waveguide. Nevertheless, there are many problems where both the characteristics of the geometry and the properties of the materials lead to very sharp solutions, with big variations throughout the domain or even field singularities in some zones. In this case, a uniform mesh or the use of linear basis functions is highly inefficient since the mesh has unknowns in zones where they are not needed, due to little variation of the solution, and lack of degrees of freedom where the variation is bigger. Increasing the mesh density or the order of the basis functions in a homogeneous way leads to better accuracy, but this is not a proper procedure since it considerably increases the computational cost of the problem, mainly in solving the algebraic problem. That is,

Manuscript received February 5, 2002; revised October 30, 2002.

A. B. Díaz-Morcillo is with the Department of Information Technologies and Communications, Polytechnic University of Cartagena, Cartagena, Murcia 30202, Spain (e-mail: alejandro.diaz@upct.es).

J. V. Balbastre and L. Nuño are with the Department of Communications, Polytechnic University of Valencia, Valencia 46022, Spain (e-mail: jbalbast@dcom.upv.es; lnuno@dcom.upv.es).

Digital Object Identifier 10.1109/TMTT.2003.810149 the mesh efficiency keeps constant if its density or the order of basis functions is increased in a uniform way.

The generation of an efficient discretization is obtained by fitting it to the distribution of the problem solution. It is possible, with the help of a friendly mesh generation tool, to create a mesh ad hoc manually or to use uniform meshes plus singular elements [1], [2] in the proximity of the singularities. Both procedures require, however, previous experience of the user in that kind of problem for finding out where a larger density of degrees of freedom or singular elements will be necessary. These drawbacks can be avoided with adaptive procedures, which can generate automatically efficient meshes that join a high accuracy and a reduced amount of degrees of freedom.

Roughly, an adaptive FEM is an iterative variant of the FEM where, in a first step, an initial mesh with few and low-order elements is generated, the corresponding algebraic problem is solved, and the error in the solution is estimated in order to add degrees of freedom in those regions of the domain with the biggest error estimation. This process is repeated until an ending condition is reached. This condition can be, for instance, a predetermined accuracy or a maximum number of degrees of freedom. Two main stages of the adaptive method can be clearly distinguished: the error indication at each element, and the selection of the regions or elements that must be refined, plus the increasing of the degrees of freedom in the selected elements, by means of an $h-, p$ - or $h p$-refinement. The so-named $r$-refinement is not included here because it consists of a repositioning of the unknowns of the problem and, although it can improve the accuracy, it is not actually an enrichment procedure.

In this paper, a new error indicator based on the recovery of the curl of the FEM solution in the modal analysis of waveguiding structures is presented and the results are discussed and compared with two previously developed error indicators. The analysis of waveguiding structures is a realistic problem that can be solved in two dimensions due to the axial symmetry of transmission lines. This kind of problem has been chosen for this study because of the following main reasons.

- It is a closed problem where the FEM can be used alone without any error component due to the truncation of the boundary.

- It leads to the solving of a generalized eigensystem and, therefore, an optimal distribution of unknowns is a key feature for reducing the computational cost of the problem.

- The visualization of the meshes generated and their main characteristics (size, shape, and order of elements) is 
easier than in a three-dimensional (3-D) problem, where the volumetric mesh make it impossible to see inner regions.

\section{Curl-ReCOVERY ERROR INDICATOR}

The error in the FEM solution of a generic vector problem is defined by

$$
\vec{e}=\vec{u}-\vec{u}_{h}
$$

where $\vec{u}$ is the exact solution and $\vec{u}_{h}$ is that obtained by means of the FEM.

For each element in the mesh, (1) becomes

$$
\vec{e}^{e}=\vec{u}^{e}-\vec{u}_{h}^{e} \text {. }
$$

The norm of this vector is a measure of the error committed at the element

$$
\left\|\vec{e}^{e}\right\|=\left\|\vec{u}^{e}-\vec{u}_{h}^{e}\right\|
$$

The total error in the problem is the sum of the elemental contributions

$$
\|\vec{e}\|^{2}=\sum_{e=1}^{n_{e}}\left\|\vec{e}^{e}\right\|^{2}
$$

Obviously, the exact solution is unknown and, therefore, it is impossible to know the exact error or the exact accuracy. However, it is possible to obtain an estimation.

Error indicators provide a reliable way to select those elements that need to be refined. They can be classified in a priori and a posteriori error indicators. Due to the lack of data related to the nature and behavior of the problem and its solution, it is very difficult to perform an a priori estimation, i.e., before the FEM solution is obtained. Therefore, most of the error indicators employed are a posteriori estimators, i.e., they calculate the error after the solution has been obtained. A reliable error estimator must assure the correct adaptation of the mesh, independently of the nature of the problem and the type of materials it presents. A great variety of error estimators can be found in the references. In [3]-[6], cursory reviews of the most-used estimators can be found. The most complete classification is in [7].

In the Zienkiewicz-Zhu or recovery error indicators, very common in civil-engineering problems, the exact solution of the problem in (1) is substituted by a recovered or smoothed solution obtained from the FEM solution, and it is assumed that this new solution is more accurate than the initial one. There are several ways for obtaining the recovered solution. Here, a new error indicator based on the patch recovery technique is presented. This technique can be applied on the same solution or on some variable related with it. In this study, the chosen variable is the vector field

$$
\vec{\sigma}=\nabla \times \vec{u}=\sigma_{x} \hat{x}+\sigma_{y} \hat{y}+\sigma_{z} \hat{z} .
$$

Taking into account that linear vector curl-conforming/scalar Lagrange basis functions in triangular elements are used, the components of the FEM solution are

$$
\begin{aligned}
& u_{x}^{e}=\sum_{i=1}^{3} \bar{u}_{i}^{e} \frac{l_{i}}{\Delta_{\varsigma}}\left[a_{i+\varsigma_{i}}-a_{i} \varsigma_{i+}\right] \\
& u_{y}^{e}=\sum_{i=1}^{3} \bar{u}_{i}^{e} \frac{l_{i}}{\Delta_{\varsigma}}\left[b_{i+\varsigma_{i}}-b_{i} \varsigma_{i+}\right] \\
& u_{z}^{e}=\sum_{i=1}^{3} \dot{u}_{i}^{e} \varsigma_{i}
\end{aligned}
$$

where $\varsigma_{i}$ are the simplex coordinates, $\dot{u}_{i}^{e}$ and $\bar{u}_{i}^{e}$ are the nodal and edge values of the solution, $l_{i}$ is the longitude of the edge $i$, and $a_{i}, b_{i}$, and $\Delta_{\varsigma}$ are given by the transformation from the real element to the canonical one, i.e.,

$$
\begin{aligned}
a_{i} & =y_{i+}-y_{i-} \\
b_{i} & =x_{i-}-x_{i+} \\
\Delta_{\varsigma} & =\left|\begin{array}{lll}
1 & x_{1} & y_{1} \\
1 & x_{2} & y_{2} \\
1 & x_{3} & y_{3}
\end{array}\right|
\end{aligned}
$$

and subscripts + and - indicate the next or previous index, respectively, in the cycle $1 \rightarrow 2 \rightarrow 3 \rightarrow 1$.

Therefore, the components of the curl will be

$$
\begin{aligned}
\sigma_{h x}^{e} & =\frac{\sum_{i=1}^{3} b_{i}^{e} \dot{u}_{h i}^{e}}{\Delta_{\varsigma}}+\gamma \sum_{i=1}^{3} V_{i}^{e} \bar{u}_{h i}^{e} \\
& =\frac{1}{\Delta_{\varsigma}}\left(\sum_{i=1}^{3} b_{i}^{e} \dot{u}_{h i}^{e}+\gamma \sum_{i=1}^{3} l_{i}\left(b_{i+}^{e} \varsigma_{i}^{e}-b_{i}^{e} \varsigma_{i+}^{e}\right) \bar{u}_{h i}^{e}\right) \\
\sigma_{h y}^{e} & =-\frac{\sum_{i=1}^{3} a_{i}^{e} \dot{u}_{h i}^{e}}{\Delta_{\varsigma}}-\gamma \sum_{i=1}^{3} U_{i}^{e} \bar{u}_{h i}^{e} \\
& =\frac{1}{\Delta_{\varsigma}}\left(\sum_{i=1}^{3} a_{i}^{e} \dot{u}_{h i}^{e}-\gamma \sum_{i=1}^{3} l_{i}\left(a_{i+}^{e} \varsigma_{i}^{e}-a_{i}^{e} \varsigma_{i+}^{e}\right) \bar{u}_{h i}^{e}\right) \\
\sigma_{h z}^{e} & =\frac{2}{\left(\Delta_{\varsigma}\right)^{2}} \sum_{i=1}^{3} l_{i}^{e}\left(b_{i+}^{e} a_{i}^{e}-b_{i}^{e} a_{i+}^{e}\right) \bar{u}_{h i}^{e}
\end{aligned}
$$

where $\gamma$ is the propagation constant.

Let

$$
\vec{e}^{e}=\vec{\sigma}_{r}^{e}-\vec{\sigma}_{h}^{e}
$$

be a measure of the elemental error, where $\vec{\sigma}_{r}^{e}$ is the recovered curl, obtained by means of a linear interpolation from the values of $\vec{\sigma}_{h}^{e}$ at the barycenters of the elements that share the central node of the patch, as shown in Fig. 1. Intuitively, it can be assumed that the recovered curl $\vec{\sigma}_{r}^{e}$ is more accurate than that obtained directly from the FEM solution because the interpolation performed on the recovered values is linear at each element and continuous throughout the domain of the problem.

Let $i$ be a node of the problem discretization and $I_{i}$ be the associated patch with $n_{i}$ elements. In Fig. 1, it can be observed that a node can have so many values at each component of $\vec{\sigma}_{h}$ as 


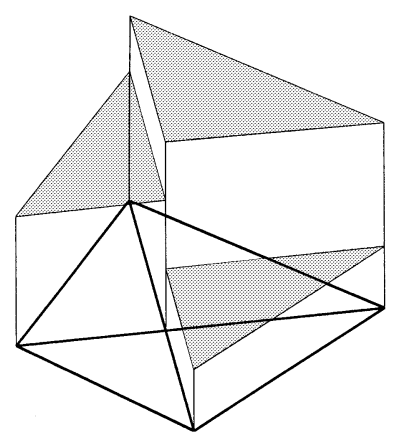

(a)

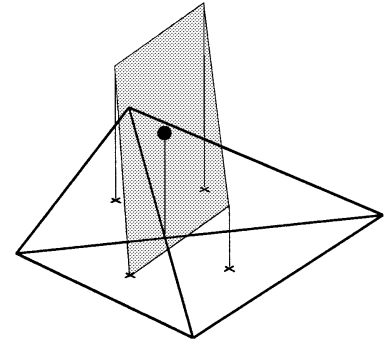

(b)

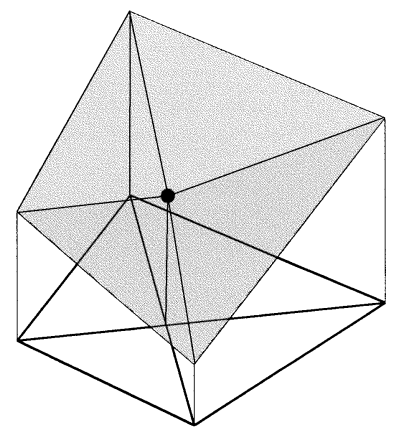

(c)
Fig. 1. Recovering procedure. (a) $\sigma_{h}^{e}$. (b) Recovered value of $\sigma_{p i}$ at the common node. (c) $\sigma_{r}^{e}$ at each element.

elements that share it. This implies a discontinuity throughout the domain. The value of $\vec{\sigma}_{h}^{e}$ must be calculated in a point with a superconvergence property, where superconvergence means the quality of decrease in the norm of the error (for the linear element) as $O\left(N^{-(1 / 2)(1+\delta)}\right)$ [8], where $N$ is the number of degrees of freedom in the problem and $\delta>0$; i.e., a convergence at least as that obtained with uniform meshes in problems with smooth solutions. In the case of the linear triangle, the superconvergence point is its barycenter.

From the $n_{i}$ values of $\vec{\sigma}_{h}^{e}$, the recovered value in the patch can be obtained for each component

$$
\sigma_{p}=c_{0}^{p}+c_{1}^{p} x+c_{2}^{p} y=\vec{P} \cdot \vec{c}^{p T}
$$

with

$$
\begin{aligned}
\vec{P}^{T} & =\left(\begin{array}{lll}
1 & x & y
\end{array}\right) \\
\vec{c}^{p T} & =\left(\begin{array}{lll}
c_{0}^{p} & c_{1}^{p} & c_{2}^{p}
\end{array}\right) .
\end{aligned}
$$

The vector $\vec{c}^{p}$ is obtained by means of least mean square fitting to the plane $\sigma_{p}$ on the values at the sample points (barycenters) for each component of the curl, i.e., the function

$$
\begin{aligned}
F^{p}\left(\vec{c}^{p}\right) & =\sum_{i=1}^{n_{j}}\left(\sigma_{h i}\left(x_{i}, y_{i}\right)-\sigma_{p i}\left(x_{i}, y_{i}\right)\right)^{2} \\
& =\sum_{i=1}^{n_{j}}\left(\sigma_{h i}\left(x_{i}, y_{i}\right)-\vec{P}\left(x_{i}, y_{i}\right) \vec{c}^{p T}\right)^{2}
\end{aligned}
$$

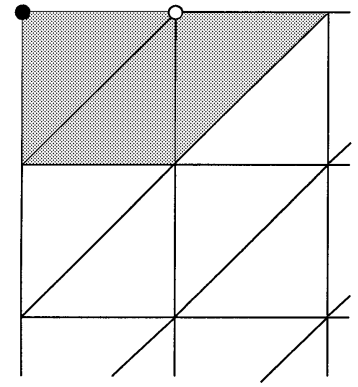

(a)

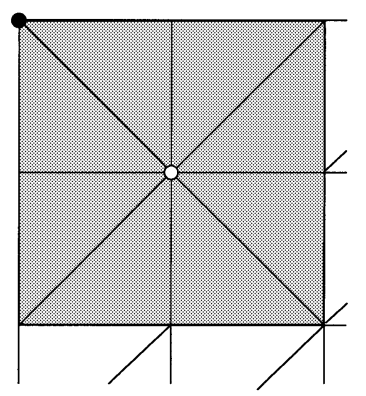

(b)

Fig. 2. Treatment of nodes belonging to less than three elements. (a) One element. (b) Two elements.

must be minimum. Therefore, deriving $F^{p}$ in regard to $\vec{c}^{p}$ and equaling to zero

$$
\sum_{i=1}^{n_{j}} \vec{P}\left(x_{i}, y_{i}\right) \vec{P}^{T}\left(x_{i}, y_{i}\right) \vec{c}^{p}=\sum_{i=1}^{n_{j}} \vec{P}\left(x_{i}, y_{i}\right) \sigma_{h i}\left(x_{i}, y_{i}\right) .
$$

The solution of this system is

$$
\vec{c}^{p}=A^{-1} \vec{b}
$$

where

$$
\begin{aligned}
A & =\sum_{i=1}^{n_{j}} \vec{P}\left(x_{i}, y_{i}\right) \vec{P}^{T}\left(x_{i}, y_{i}\right) \\
\vec{b} & =\sum_{i=1}^{n_{j}} \vec{P}\left(x_{i}, y_{i}\right) \sigma_{h i}\left(x_{i}, y_{i}\right) .
\end{aligned}
$$

For solving (20), it is required that the patch has, at least, three elements, since three points (values of $\sigma_{h}$ ) are needed for defining the plane $\vec{c}^{p}$. However, in the mesh, concretely at its boundary, there can be nodes that belong only to one or two elements, as shown in Fig. 2 (the existence of, at least, three elements in the patches of inner nodes is guaranteed by the connectivity of the mesh). In those cases, the value assigned to those nodes (black points in Fig. 2) is extrapolated from the function $\sigma_{p}$ of the patch of a neighboring node (white point). In a triangular mesh, it is guaranteed that a node belonging to one or two elements will have always a neighboring node that belongs to three or more elements, excepting, of course, the trivial case of a mesh with only two elements.

Once the expression of $\sigma_{p}$ is known, it is possible to obtain the value at the central node. When repeating this operation in all the nodes in the mesh, the smoothed value of the curl is obtained. This recovered value $\sigma_{r}^{e}$, which is used as the more accurate 
solution, has been obtained here by means of a nodal Lagrange interpolation from $d e$ values of $\sigma_{p}$ at each node of the triangle [see Fig. 1(c)].

Once $\vec{\sigma}_{r}^{e}$ has been obtained, the error indicator is calculated as the norm $L_{2}$ of $\vec{e}$ as follows:

$$
\begin{aligned}
\left\|\vec{e}^{e}\right\|^{2}= & \iint_{\Omega^{e}} \vec{e} e^{*} \cdot \vec{e}^{e} d \Omega \\
= & \iint_{\Omega^{e}}\left|\sigma_{r x}^{e}-\sigma_{h x}^{e}\right|^{2} d \Omega \\
& +\iint_{\Omega^{e}}\left|\sigma_{r y}^{e}-\sigma_{h y}^{e}\right|^{2} d \Omega+\iint_{\Omega^{e}}\left|\sigma_{r z}^{e}-\sigma_{h z}^{e}\right|^{2} d \Omega
\end{aligned}
$$

and the use of linear basis functions allows an analytical integration.

It is important to remark that, if the dual field has been obtained in the problem, the curl (5) at each element is already known, and the computational cost of this error indicator is minimum. However, the residual error indicator requires the calculation of $\nabla \times \nu_{e}^{-1} \nabla \times \vec{u}^{e}$, where $\nu_{e}^{-1}$ is the magnetic permeability or electric permittivity (depending on the formulation of the problem) for obtaining the inner residual. For linear basis functions, the computational cost of this operation is negligible, but it increases with the order of the polynomial functions.

The generalization of the curl-recovery error indicator to higher order basis functions is straightforward: for $p$-order basis functions, the fitting of the curl should be done to a $p$-order surface.

\section{MESH REFINEMENT}

In an adaptive procedure, the stage of mesh refinement consists of the addition of degrees of freedom in those regions where the problem has a poor discretization, i.e., where the error is bigger. The goal of this refinement is obtaining, in the last step of refinement, a nearly homogeneous distribution of the error in the mesh. For performing the refinement, a criterion for the selection of the elements and a way for adding new degrees of freedom must be defined.

\section{A. Selection of Refinement Regions}

In most of the error indicators, the error is element associated. Thus, the obvious criterion is choosing those elements that have an error larger that a given value. This value can be the root mean square estimated error per element

$$
\|e\|_{m}=\frac{\|e\|_{T}}{\sqrt{n_{e}}} .
$$

Thus, the elements with $\left\|e_{e}\right\|>\|e\|_{m}$ are refined and the error in the mesh becomes more uniform. Another possibility is comparing the error at each element with a fraction of the maximum error in the mesh. In this case, the chosen elements are those with

$$
\left\|e_{e}\right\|>\kappa\|e\|_{\max }
$$

where $\kappa(0<\kappa<1)$ is a parameter that controls the amount of refined elements and, therefore, the amount of new degrees of freedom generated at each step of the adaptive process. For large $\kappa$, only those elements with an error close to the maximum are refined, and this leads to many steps in the adaptation process for obtaining a good accuracy. On the other hand, values close to zero generate an excessive amount of new unknowns that do not improve the accuracy, but increase the computational cost. A widely used value for this parameter is 0.5 . Other criteria can be added to this, for instance, a maximum and minimum number of refined elements at each step [9].

\section{B. Refinement Techniques}

Once the elements to be refined are known, different strategies can be defined for adding the new unknowns. The most used strategy is the simple refinement, where all the chosen elements for refinement are refined in the same way. Other strategies are the multiple refinement, where several levels of refinement are defined [10]-[12] and the so-called forward-backward refinement [13], [14], where the initial mesh has a medium density and, at each step of adaptation, new unknowns can be added in the regions with large error and some unknowns can be eliminated where the error is small.

A mesh can increase its degrees of freedom in two ways: splitting some elements in smaller ones or increasing the order of the basis functions in some elements. The first one is known as $h$-refinement and the second one as $p$-refinement [15], [16]. A third alternative is a combination of both techniques, the so-named $h p$-refinement. Some examples of this strategy can be found in [17]-[19].

The main difficulty of an $h$-type refinement is the generation of transition elements that assure the conformity of the mesh. In this study, in a first step, the chosen elements for refinement are split in four elements following a regular refinement $1: 4$. This refinement guarantees the same aspect ratio in the new elements or, if an edge exchange criterion is performed, even better aspect ratios in some new elements [20]. In a second step, the nonconformity situations are solved by means of generation of transition elements [6]. It is important to remark that, following this strategy, the aspect ratio of the elements throughout the adaptive process is bounded. Concretely, the smallest angle is, at least, one-half of the smallest angle in the initial mesh. After the splitting, a heredity mechanism is necessary because the new nodes, edges, and elements must have the same properties (boundary conditions or material properties) as their parents.

In a $p$-refinement, the size and number of elements do not vary during the adaptation process. The way of increasing the number of unknowns is increasing the order of the polynomial basis functions. Since polynomials of different order can be used in the same mesh, it is possible that some pairs of elements that share an edge have different basis functions and there be a discontinuity of the tangential component on the common edge. It is very difficult to eliminate this discontinuity if interpolatory basis functions [21] are employed. However, the use of hierarchical basis functions [22] provides an easy way for maintaining the tangential continuity. Since they form a hierarchical basis, functions of order $p$ are a subset of the functions of order $p+1$. Thus, the continuity is guaranteed by eliminating in the matrices of the algebraic system the row and column corresponding to the unknowns of order $p+1$ on the shared edge. 


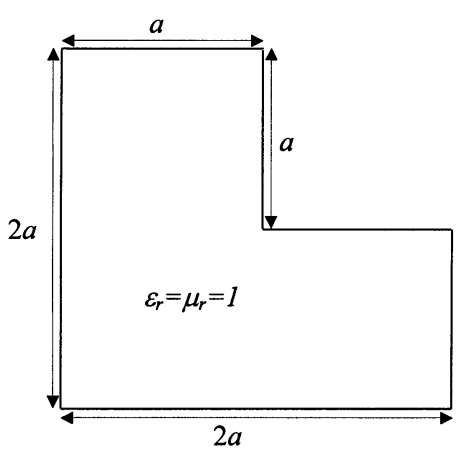

(a)

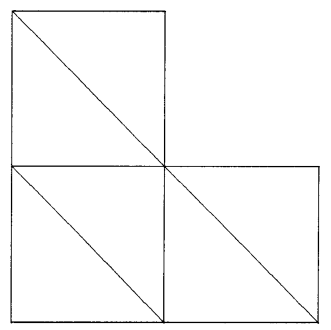

(b)

Fig. 3. L-shaped waveguide. (a) Structure. (b) Initial mesh.

\section{RESUlTS AND DISCUSSION}

In this study, the first mode in different waveguiding structures (an L-shaped homogeneous waveguide, a shielded unilateral finline, and a shielded coplanar line with anisotropic and lossy substrate) has been analyzed. All these structures present singularities in the solution. In those cases, an adaptive FEM procedure significantly decreases the computational cost of the problem. In all cases, an electric-field formulation and a $1: 4$ $h$-refinement with $\kappa=0.5$ have been used. For analyzing the performance of the adaptive process, the sequence of adapted meshes is displayed and the convergence curves for the curl-recovery error indicator are compared with those obtained with a complete residual error indicator [7], a flux continuity error indicator [20], and a classical FEM, i.e., that obtained when the density of the mesh is increased uniformly. The computation of the relative error in the propagation constant and the characteristic impedance requires an exact or reference value. The value obtained in an eight-step adaptive process has been employed for this purpose.

\section{A. L-Shaped Homogeneous Waveguide}

This waveguide presents a field singularity at its inner corner. Fig. 3 shows its structure and the initial mesh in the adaptation process. The results were obtained for $k_{0}=7.87 a$. The reference value, i.e., that obtained in the eighth step of the adaptive FEM, was $\beta / k_{0}=0.9242$. The phase constant obtained by means of the commercial tool MAFIA (finite-integration technique [23], 100000 nodes) was $\beta / k_{0}=0.9246$. The sequence of adapted meshes throughout the process (Fig. 4) shows that the error indicator detects properly the singularity. An excessive refinement can be observed in zones where a large error is not expected. This is due to a smaller accuracy of the error indication because, in those zones, there are nodes that belong to less than three elements and, therefore, the recovered value is obtained

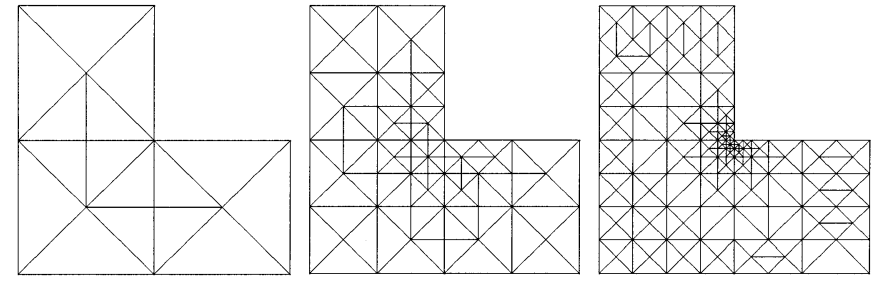

Fig. 4. Adapted meshes (first, fourth, and sixth) for the curl-recovery error indicator.

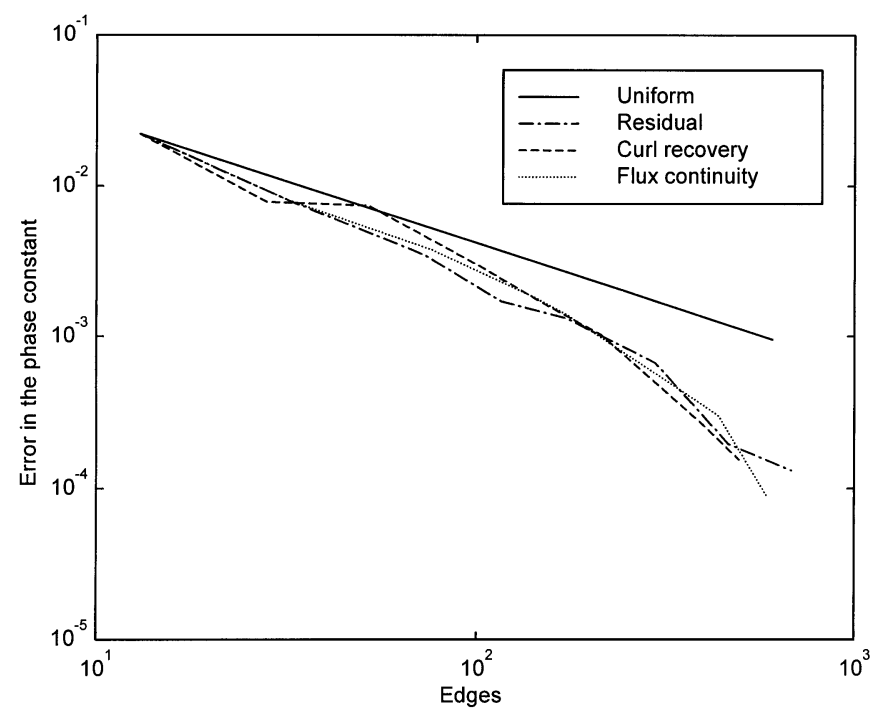

Fig. 5. Convergence for different error indicators in an L-shaped waveguide.

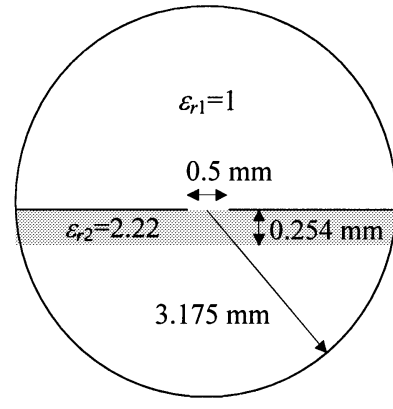

(a)

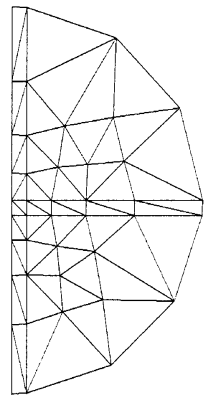

(b)

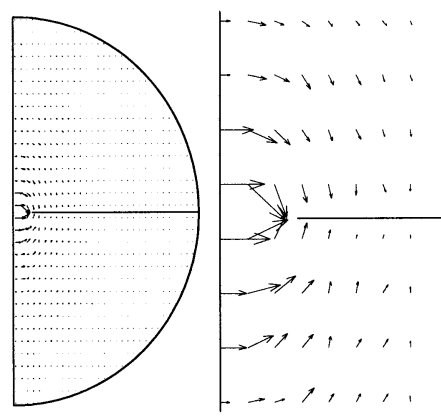

(c)

(d)

Fig. 6. Shielded unilateral finline. (a) Structure. (b) Initial mesh (one-half). (c) Transversal electric field (first mode). (d) Zoom of the singularity zone.

from an extrapolation of the value in a neighboring node. Nevertheless, that refinement is not intense and does not significantly influence the convergence of the process. As Fig. 5 shows, this 

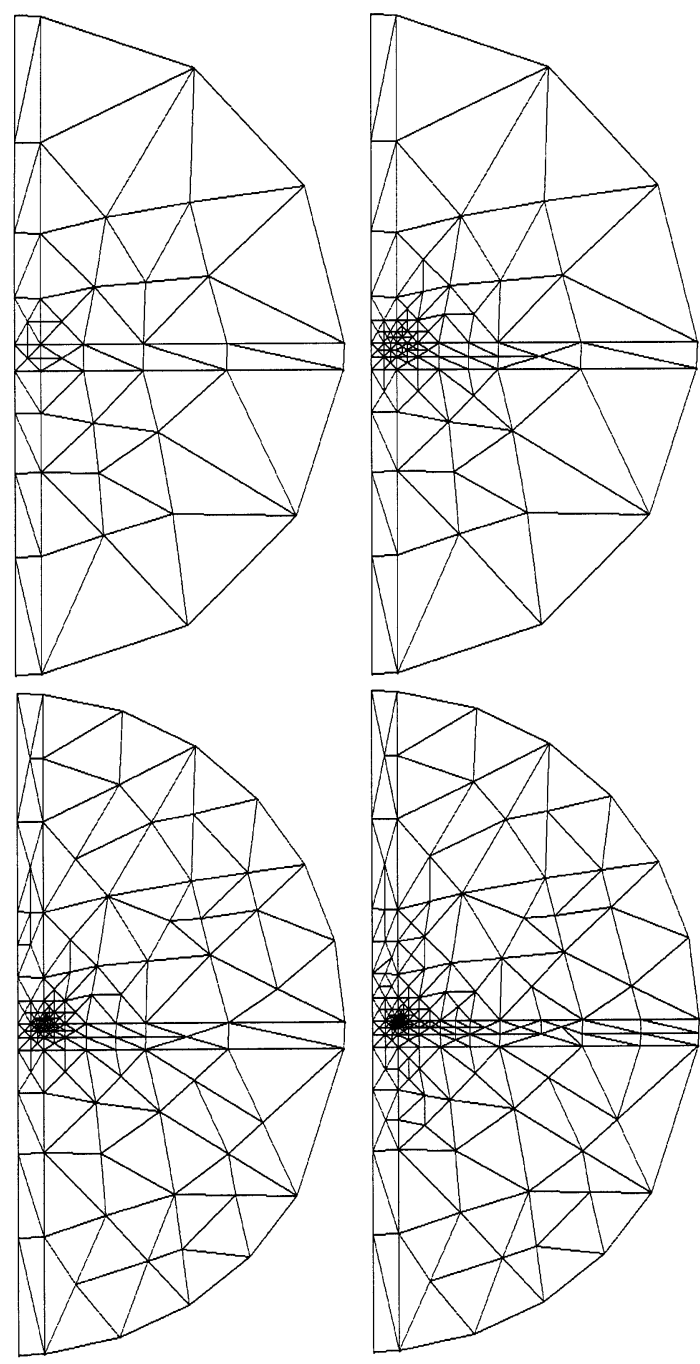

Fig. 7. Adapted meshes (first, third, fourth, and fifth) for the curl-recovery error indicator.

convergence is similar to those of the residual and flux continuity error indicators. For the wave impedance, the convergence obtained with the adaptive procedure is that of Fig. 5 since, in a TE mode, this parameter only depends on the propagation constant

$$
Z_{\mathrm{TE}}=j \eta \frac{k_{0}}{\gamma}
$$

The computational cost of the adaptation stage (error indication plus mesh refinement) for this and the other two examples was negligible (less than $0.01 \%$ ) in regard to the computational cost of the FEM solving stage.

\section{B. Shielded Unilateral Finline}

Fig. 6 shows the structure of a cylindrical shielded unilateral finline, the initial mesh, and the transversal electric field for the first mode. Due to the symmetry of the structure, the adaptation process has been performed on one-half of the line, imposing an electric wall condition on the symmetry axis. The main characteristic of this line in regard to the adaptive process is the curved boundary. In this case, the splitting of the boundary edges, when
TABLE I

Normalized Phase Constant at 20 GHz FOR the First Mode IN THE SHIELDED UNILATERAL FINLINE

\begin{tabular}{c|c|c}
\hline Reference value (8 ${ }^{\text {th }}$ step) & MAFIA, 50.000 nodes & Wu and Vahldieck [24] \\
\hline 0.9191 & 0.9329 & 0.9638 \\
\hline
\end{tabular}

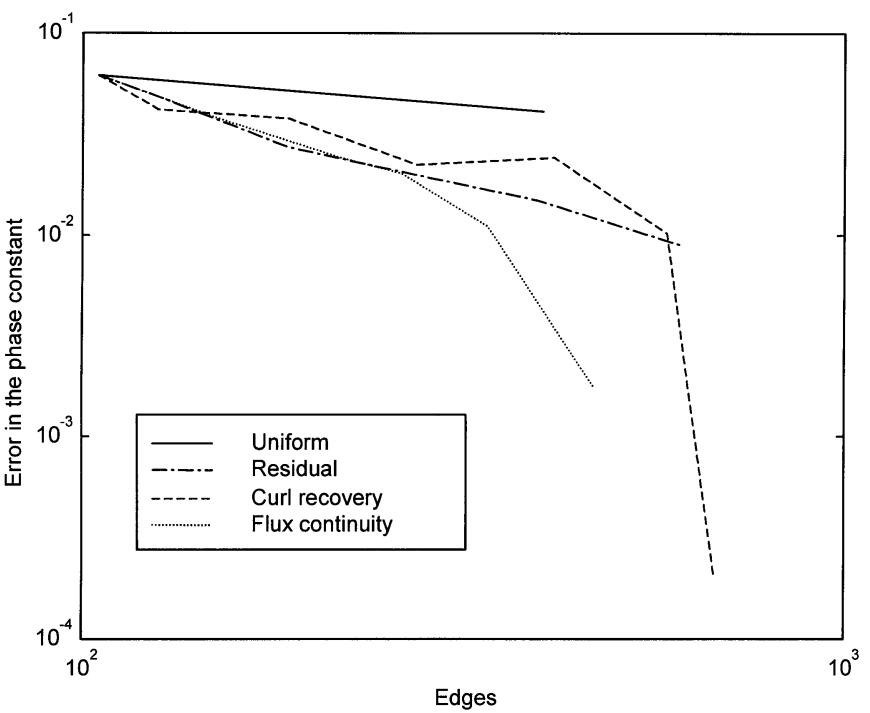

Fig. 8. Convergence of $\beta$ for different error indicators in a unilateral finline.

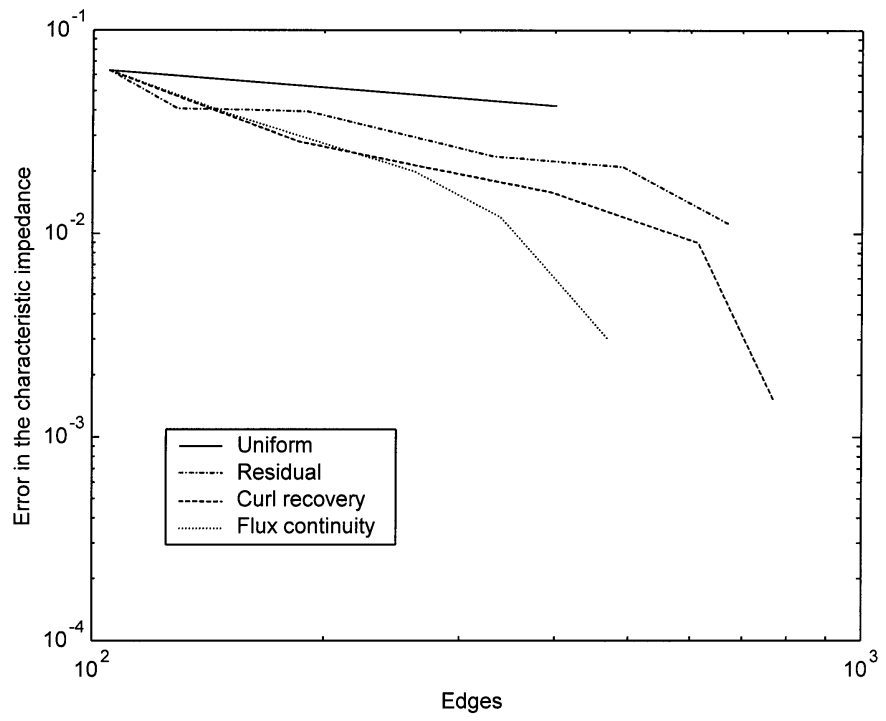

Fig. 9. Convergence of $Z_{0}$ for different error indicators in a unilateral finline.

a refinement is necessary, must take into account the curvature of the parent edge and transfer it to the children edges. This splitting modifies the boundary of the problem throughout the adaptive process. As Fig. 7 shows, the adaptation process increased the degrees of freedom around the edge of the inner zero-thickness conductor, i.e., the singularity zone. Table I compares the reference value for the phase constant at $20 \mathrm{GHz}$, obtained in an eight-step adaptive FEM, values obtained with MAFIA, and found in the literature.

For the computation of the characteristic impedance of the dominant mode in the finline, (28) was used as follows:

$$
Z_{0}=\frac{V^{2}}{2 P}
$$




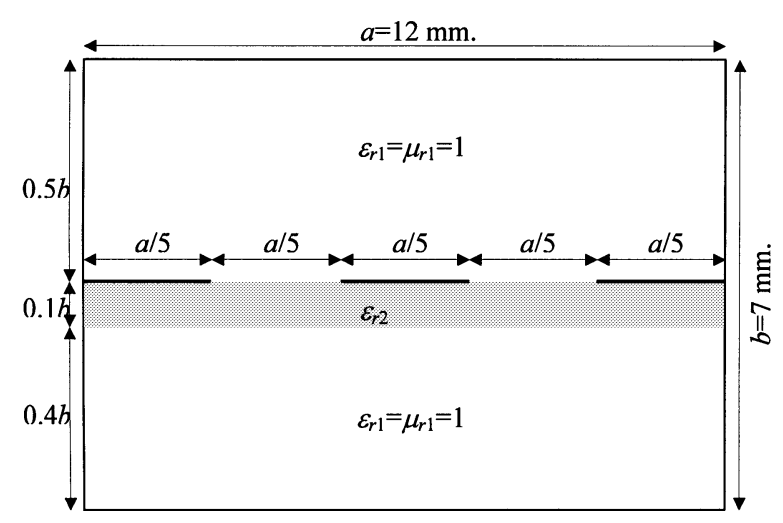

(a)

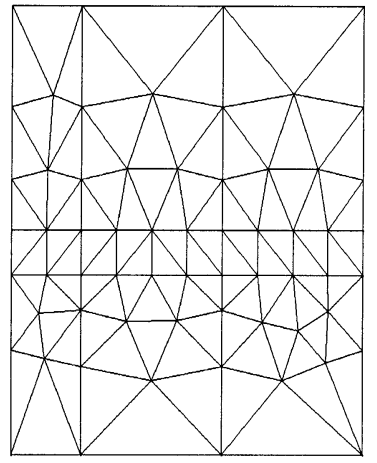

(b)

Fig. 10. Shielded coplanar line. (a) Structure. (b) Initial mesh (one-half).

where

$$
\begin{aligned}
V & =\int_{-w / 2}^{w / 2} E_{x}\left(x, y=y_{\text {strip }}\right) d x \\
P & =\frac{1}{2} \Re\left\{\iint_{\Omega}\left(\vec{E} \times \vec{H}^{*}\right) \hat{z} d s\right\} .
\end{aligned}
$$

The convergence curves (Figs. 8 and 9) of the phase constant and characteristic impedance verify the improvement in regard to convergence of the FEM with uniform or, in this case, graded meshes. The curl-recovery error indicator again has a similar convergence as the residual indicator, except at the last stage, when the curl recovery obtains a very accurate result.

\section{Shielded Coplanar Line}

Finally, the results obtained in the analysis of a shielded coplanar rectangular line are presented. This structure contains three zero-thickness metallic strips that produce singularity zones at their edges. For the sake of generality, a nonphysical lossy and anisotropic dielectric material with

$$
\varepsilon_{r 2}=\left[\begin{array}{lll}
8-j 0.2 & 4-j 0.1 & 3-j 0.1 \\
4-j 0.1 & 6-j 0.1 & 4-j 0.2 \\
3-j 0.1 & 4-j 0.2 & 7-j 0.3
\end{array}\right]
$$

was used as a substrate.

The structure and initial mesh are shown in Fig. 10. Once again, the first mode in this line has been obtained in one-half of the structure imposing, in this case, a magnetic wall condition at the symmetry axis. The first, third, and fourth meshes of the
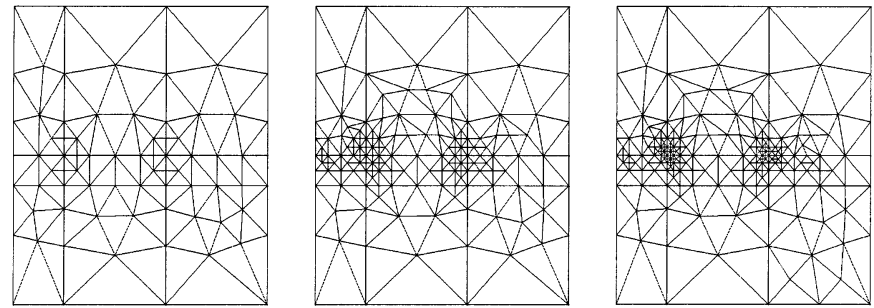

Fig. 11. Adapted meshes (first, third, and fourth) for the curl-recovery error indicator.

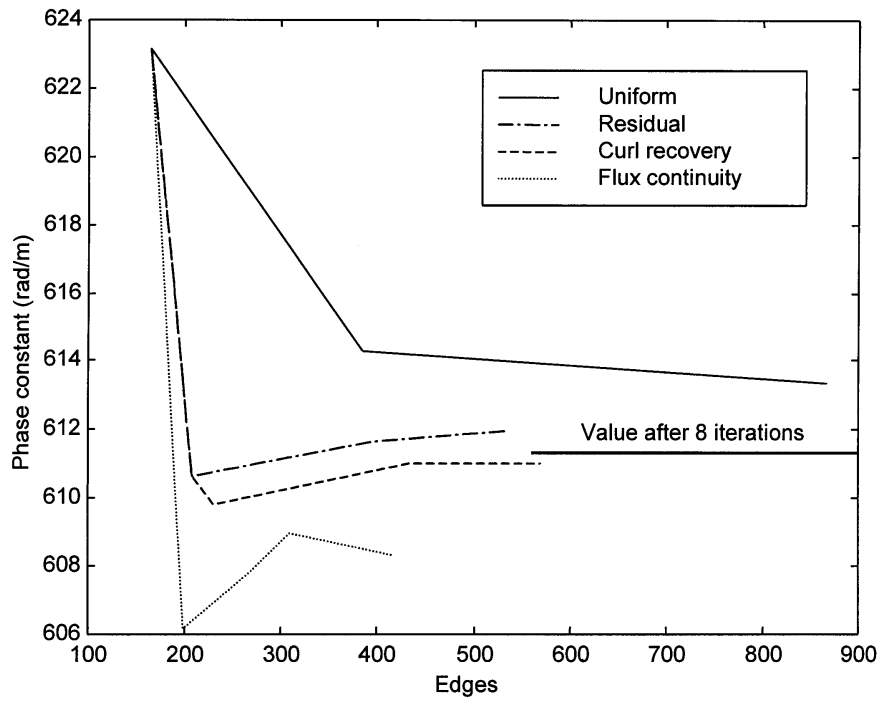

Fig. 12. Phase constant for different error indicators in a shielded coplanar line.

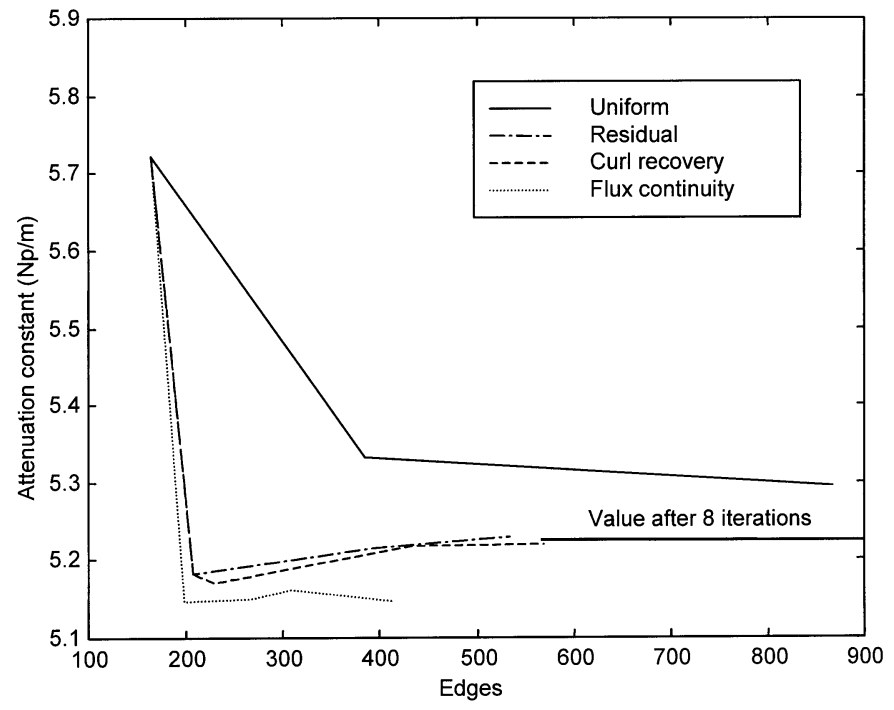

Fig. 13. Attenuation constant for different error indicators in a shielded coplanar line.

adaptive process (Fig. 11) show a correct detection of both singularities. For this example, a direct representation of the values of the propagation constant obtained at each step is printed in Figs. 12 and 13. This representation shows how these values reach the reference value obtained after eight adaptive steps. An asymptotic nonmonotonic behavior is obtained for both the phase and attenuation constants. This behavior is similar for the 
TABLE II

COMPARISON OF THE ERROR INDICATORS

\begin{tabular}{c|c|c|c}
\hline & Residual & Flux continuity & Curl recovery \\
\hline Type & Complete residual & Incomplete residual & Heuristic, patch recovery \\
\hline Computational cost & Negligible & Negligible & Negligible \\
\hline Implementation & Difficult & Easy & Easy \\
\hline Treatment of singularities & Detection & Overestimation & Detection \\
\hline
\end{tabular}

residual error indicator, but the flux continuity indicator presents a slower convergence to the exact value or even diverges in some step. This result can be explained by an ill-conditioning matrix in the generalized eigensystem that must be solved since, as a general rule, the flux continuity indicator overestimates the singularity and generates an excessive refinement, obtaining neighboring elements very different in size. This behavior is due to its incomplete residual nature, i.e., while the residual error indicator takes into account the fulfillment of the vector wave equation in the element and the boundary conditions on its edges, the flux continuity indicator only measures the boundary condition fulfillment. Table II compares the main characteristics of these three error indicators.

\section{CONCLUSIONS}

A new recovery error indicator has been developed and applied in an adaptive FEM procedure for the analysis of waveguiding structures. This error indicator, complemented with an $h$-refinement technique that maintains the triangle's aspect ratio bounded throughout the adaptive process, has obtained convergence rates much better that the classical FEM with uniform or graded meshes, and presents a better performance than the flux continuity indicator and similar or, in some cases, better than the complete residual indicator. In regard to the computational cost, the three indicators showed a negligible CPU time in front of the time for obtaining the matrices of the eigensystem and solving it. Nevertheless, if higher order basis functions are used, the curl-recovery indicator appears to be a good candidate for estimating the error due to its simpler formulation and easier implementation.

\section{REFERENCES}

[1] J. M. Gil and J. P. Webb, "A new edge element for the modeling of field singularities in transmission lines and waveguides," IEEE Trans. Microwave Theory Tech., vol. 45, pp. 2125-2130, Dec. 1997.

[2] Z. Pantic-Tanner, J. S. Savage, D. R. Tanner, and A. F. Peterson, "Twodimensional singular vector elements for finite element analysis," IEEE Trans. Microwave Theory Tech., vol. 46, pp. 178-184, Feb. 1998.

[3] P. Fernandes, P. Girdinio, P. Molfino, and M. Repetto, "Local error estimates for adaptive mesh refinement," IEEE Trans. Magn., vol. 24, pp. 299-302, Jan. 1988.

[4] E. Hinton, N. V. R. Rao, and M. Özakça, "Mesh generation with adaptive finite element analysis," Adv. Eng. Softw., vol. 13, no. 5/6, pp. 238-262, 1991.

[5] M. Ainsworth and A. Craig, "A posteriori error estimators in the finite element method," Numer. Math., vol. 60, pp. 429-463, 1992.

[6] R. Verfürth, A Review of A Posteriori Error Estimation and Adaptive Mesh-Refinement Techniques. New York: Wiley-Teubner, 1996.

[7] M. Salazar-Palma, T. K. Sarkar, L. E. García-Castillo, T. Roy, and A. Djordjevic, Iterative and Self-Adaptive Finite Elements in Electromagnetic Modeling. Norwood, MA: Artech House, 1998.
[8] R. Durán, M. A. Muschietti, and R. Rodríguez, "On the asymptotic exactness of error estimators for linear triangular finite elements," Numer. Math., vol. 59, pp. 107-127, 1991.

[9] L. Jänicke and A. Kost, "Error estimation and adaptive mesh generation in the 2D and 3D finite element method," IEEE Trans. Magn., vol. 32, pp. 1334-1337, May 1996.

[10] Z. J. Cendes and D. N. Shenton, "Adaptive mesh refinement in the finite element computation of magnetic fields," IEEE Trans. Magn., vol. MAG-21, pp. 1811-1816, Sept. 1985.

[11] P. L. Baehmann and M. S. Shephard, "Adaptive multiple-level $h$-refinement in automated finite element analysis," Eng. Comput., vol. 5, pp. $235-247,1989$.

[12] P. Fernandes, P. Girdinio, M. Repetto, and G. Secondo, "Refinement strategies in adaptive meshing," IEEE Trans. Magn., vol. 28, pp. 1739-1742, Mar. 1992.

[13] M. C. Rivara, "Local modification of meshes for adaptive and/or multigrid finite-element methods," J. Comput. Appl. Math., vol. 36, pp. 79-89, 1991.

[14] T. Belytschko and M. Tabbara, " $H$-adaptive finite element methods for dynamic problems, with emphasis on localization," Int. J. Numer. Methods Eng., vol. 36, pp. 4245-4265, 1993.

[15] J. P. S. R. Gago, D. W. Kelly, and O. C. Zienkiewicz, "A posteriori error analysis and adaptive processes in the finite element method: Part II-Adaptive mesh refinement," Int. J. Numer. Methods Eng., vol. 19, pp. 1621-1656, 1983.

[16] S. McFee and J. P. Webb, "Adaptive finite element analysis of microwave and optical devices using hierarchal triangles," IEEE Trans. Magn., vol. 28, pp. 1708-1711, Mar. 1992.

[17] O. C. Zienkiewicz, J. Z. Zhu, and N. G. Gong, "Effective and practical $h-p$ version adaptive analysis procedures for the finite element method," Int. J. Numer. Methods Eng., vol. 28, pp. 879-891, 1989.

[18] M. Ainsworth and J. T. Oden, "A procedure for a posteriori error estimation for $h-p$ finite element methods," Comput. Methods Appl. Mech. Eng., vol. 101, pp. 73-96, 1992.

[19] D. Giannacopoulos and S. McFee, "Toward optimal $h-p$ adaption near singularities in finite element electromagnetics," IEEE Trans. Magn., vol. 30, pp. 3523-3526, Sept. 1994.

[20] A. Díaz-Morcillo, L. Nuño, and J. V. Balbastre, "A new error indicator for the analysis of waveguiding structures by the adaptive finite element method," Microwave Opt. Technol. Lett., vol. 27, pp. 361-366, Dec. 2000.

[21] R. D. Graglia, D. R. Wilton, and A. F. Peterson, "Higher order interpolatory vector bases for computational electromagnetics," IEEE Trans. Antennas Propagat., vol. 45, pp. 329-342, Mar. 1997.

[22] J. P. Webb, "Hierarchal vector basis functions of arbitrary order for triangular and tetrahedral finite elements," IEEE Trans. Antennas Propagat., vol. 47, pp. 1244-1253, Aug. 1999.

[23] J. E. Lebaric and D. Kajfez, "Analysis of dielectric resonator cavities using the finite integration technique," IEEE Trans. Microwave Theory Tech., vol. 37, pp. 1740-1748, Nov. 1989.

[24] K. Wu and R. Vahldieck, "The method of lines applied to planar transmission lines in circular and elliptic waveguides," IEEE Trans. Microwave Theory Tech., vol. 37, pp. 1958-1963, Dec. 1989.

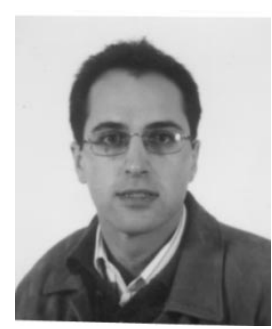

Alejandro B. Díaz-Morcillo (S'95-M'02) was born in Albacete, Spain, in 1971. He received the Ingeniero (Ms.Eng.) and Doctor Ingeniero (Ph.D.) degrees in telecommunication engineering from the Polytechnic University of Valencia (UPV), Valencia, Spain, in 1995 and 2000, respectively.

From 1996 to 1999, he was a Research Assistant with the Department of Communications, UPV. In 1999, he joined the Department of Information Technologies and Communications, Polytechnic University of Cartagena (UPCT), Cartagena, Spain, as Teaching Assistant, and where, since 2001, he has been an Associate Professor. His main research interests are numerical methods in electromagnetics and industrial microwave heating systems. 
Juan V. Balbastre (S'90-A'96-M'97) was born in Mislata, Spain, in 1969. $\mathrm{He}$ received the Ingeniero (Ms.Eng.) and Doctor Ingeniero (Ph.D.) degrees in telecommunication engineering from the Polytechnic University of Valencia (UPV), Valencia, Spain, in 1993 and 1996, respectively.

In 1993, he joined the Department of Communications, UPV, as Teaching Assistant, and where, since 1998, he has been an Associate Professor. His main research interests are numerical methods, electromagnetic compatibility, and industrial microwave heating systems.

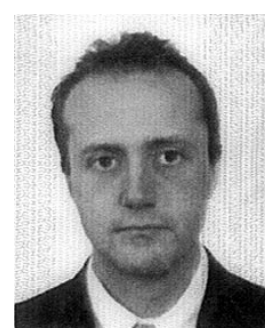

Luis Nuño (M'95) was born in Valencia, Spain, in 1963. He received the Ingeniero (Ms.Eng.) degree in telecommunication engineering from the Polytechnic University of Madrid (UPM), Madrid, Spain, in 1987, and the Doctor Ingeniero (Ph.D.) degree in telecommunication engineering from the Polytechnic University of Valencia (UPV), Valencia, Spain, in 1993.

From 1986 to 1988 , he was with the Grupo de Radiación, UPM, and from 1988 to 1990 , he was with the enterprise Ingeniería de Radiofrecuencias (IRSA). Since 1990, he has been with the Department of Communications, UPV, where he became a Teaching Assistant in 1990, Associate Professor in 1996, and Professor in 2002. He is also the responsible for the Electromagnetic Compatibility (EMC) Laboratory (ICEM), UPV, since its creation in 1992. His current research interest include numerical methods in electromagnetism, electromagnetic compatibility, and industrial applications of microwaves. 